\title{
Probiotics in Periodontics Good or Bad!!! A Review
}

\author{
Dr. Ranjeeta Dham ${ }^{1}$, Dr. Sharnamma B ${ }^{2}$, Dr. Poonam Dutt ${ }^{3}$, Dr. Prashant Tyagi ${ }^{4}$ \\ ${ }^{1}$ (P.G Student,Dept. of Periodontics, Shree Bankey Bihari Dental College, Ghaziabad,India) \\ ${ }^{2}$ (Professor and Head, Dept of Periodontics,Shree Bankey Bihari Dental College, Ghaziabad,India) \\ ${ }_{3}^{3}$ (Professor, Dept of Periodontics, Shree Bankey Bihari Dental College, Ghaziabad,India) \\ ${ }^{4}$ (Sr.Lecturer, Dept .of Periodontics,Shree Bankey Bihari Dental College, Ghaziabad,India)
}

\begin{abstract}
Probiotics are non-pathogenic living micro-organisms which have been added to various foods because of their beneficial effects for human health. There has been a paradigm shift of treatment from specific bacterial elimination to altering bacterial ecology by probiotics. With the increase in the incidence of résistance to antibiotics, probiotics may be a promising area in research in periodontal therapy. They play a beneficial role in preventing common oral health problems such as dental carries, periodontal disease, fungal Infections (Candida) and halitosis. A complete understanding of the broad ecologic changes in the mouth is essential to assess their long term consequences of oral health and disease. The paper reviews the evidence for the use of probiotics and prebiotics for maintenance of oral health and for the prevention of periodontal disease.
\end{abstract}

Keywords: Probiotics, Prebiotics, Dental Caries, Halitosis, Oral Health, Periodontal Disease

\section{Introduction}

Probiotics can be defined as living microbes, or a food ingredient containing living microbes, that beneficially influence the health of the host when used in adequate numbers.

The intestine normal micro-flora is metabolically active but as yet unexpected organ of host defence. The large intestine contains 300-500 species of bacteria. ${ }^{1}$ Some of these are potential pathogens and cause infection under certain circumstances, and it has been proved that probiotics and prebiotics have an impact or gut flora, and most clinical interest has focused on the prevention or treatment of gastro-interval infections and disease. $^{2}$

Probiotics for oral health are not that prolific. The term probiotics seems innocuous enough. The term may be little misleading because all probiotics are taken by mouth, they have rarely administered via injections, enema or calories. Probiotics almost all functions in the gut, though. The oral probiotics of their article refer to those bacteria that are intended to work in the mouth to alter the oral biofilm to be more healthful. Yes, it is possible to have a more healthful biofilm.

The original observation of the positive role played by some selected bacteria was scientifically investigated by Elimetchuikoff, the Ukrainian- born Nobel Prize winner working at the Pasteur Institute at the beginning of the last century. He proposed, in 1907, that the lactic acid producing strain lactobacillus bulgarius (containing in Bulgarian Yogurt) is able displace pathological intestinal micro-biota. The term "Probiotics" the antonym of the term "antibiotics" was introduced in 1965 by Lilly and Stillwell as substances produced by micro-organisms which promote the growth of other micro-organisms. They showed that several species of protozoa, during their lagaithmic phases of growth, produced substances that prolong the logarithmic phase in other species. $^{3}$

The importance of living cells in probiotics was emphasized by Fuller in 1989, who defined probiotics as a "A live microbial feed supplement which beneficially affects the host animals by improving its intestinal microbial balance. ${ }^{4}$ Since several definitions of probiotics have been proposed as shown in (tab 1). The currently used consensus definition of probiotics was put forward by the world health organization and by the Food and Agriculture organizations of the United States. They defined Probiotics as "Live Micro-organisms" which when administrated in adequate amounts confer a health benefit on the host. ${ }^{4}$ 
Table No.1 Definitions of Probiotics

\begin{tabular}{|c|c|c|}
\hline Year & Definitions & Reference \\
\hline 1965 & $\begin{array}{l}\text { Substances produced by micro-organisms that produce the } \\
\text { growth of other micro - organisms }\end{array}$ & Lilly and Stillwell \\
\hline 1974 & $\begin{array}{l}\text { Organisms and substances that contribute to intestinal } \\
\text { microbial balance }\end{array}$ & Parker \\
\hline 1989 & $\begin{array}{l}\text { A live microbial feed supplement that beneficially affects the } \\
\text { host animal by improving its intestinal microbial balance. }\end{array}$ & Fuller \\
\hline 1992 & $\begin{array}{l}\text { A viable monoculture or mixed-culture of micro-organisms } \\
\text { that, when applied to animal or human, beneficially affects the } \\
\text { host by improving the properties of the indigenous micro- } \\
\text { flora. }\end{array}$ & Havenaar and Huis Int Veld \\
\hline 1996 & $\begin{array}{l}\text { Living micro-organisms that, upon ingestion in certain } \\
\text { numbers, exert health benefits beyond inherent basic nutrition. }\end{array}$ & Schaafsma \\
\hline 1999 & $\begin{array}{l}\text { A microbial dietary adjuvant that beneficially affects the host } \\
\text { physiology by modeling mucosal and systemic immunity, as } \\
\text { well as by improving nutritional and microbial balance in the } \\
\text { intestinal tract. }\end{array}$ & Naidu at al. \\
\hline 2001 & $\begin{array}{l}\text { A preparation of, or a product containing viable, defined } \\
\text { microorganism In sufficient numbers, which alter the } \\
\text { microflora (by implantation or colonization)in a compartment } \\
\text { of the host and as such exert beneficial health effects in this } \\
\text { host. }\end{array}$ & Schrezemair And De Vrese \\
\hline 2001 & $\begin{array}{l}\text { Live micro-organisms that, when } \mathrm{FAO} / \mathrm{WHO} \text { administered in } \\
\text { adequate amounts, confer a health benefit to the host. }\end{array}$ & FAQ/WHO report \\
\hline
\end{tabular}

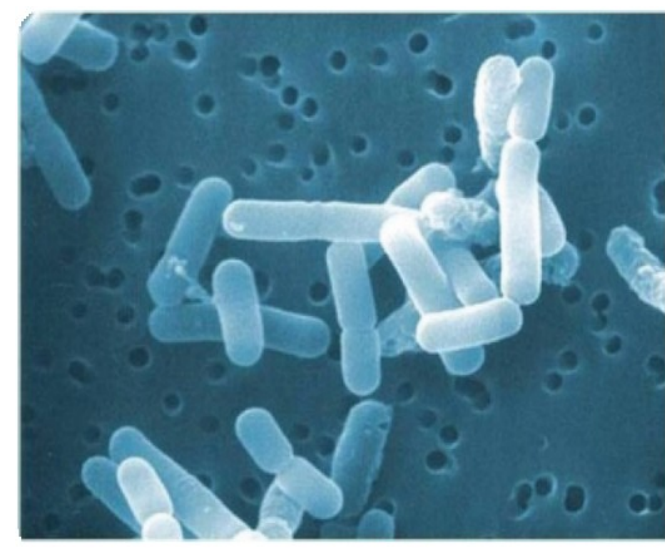

Fig: 1

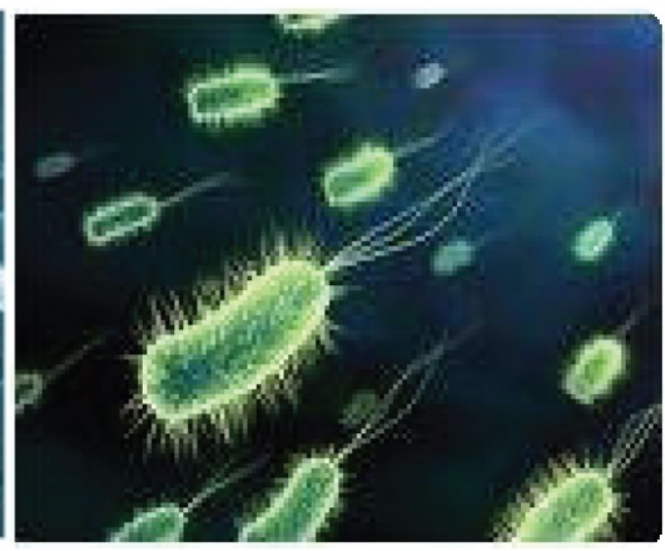

Fig: 2

\section{A MUTUALLY BENEFICIAL RELATIONSHIP BETWEEN BACTERIA AND HOST (HUMAN BEING)}

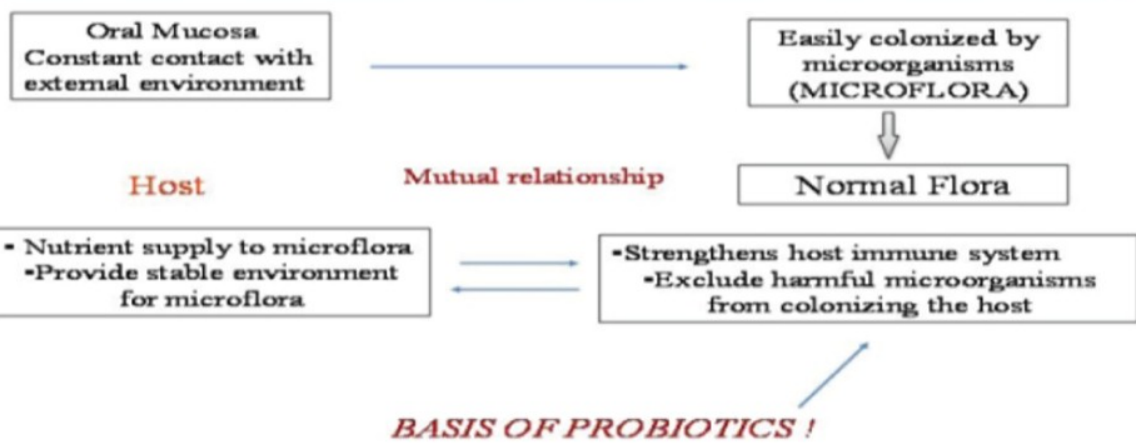

Fig 3 


\section{Oral Cavity -A Dynamic Ecosystem}

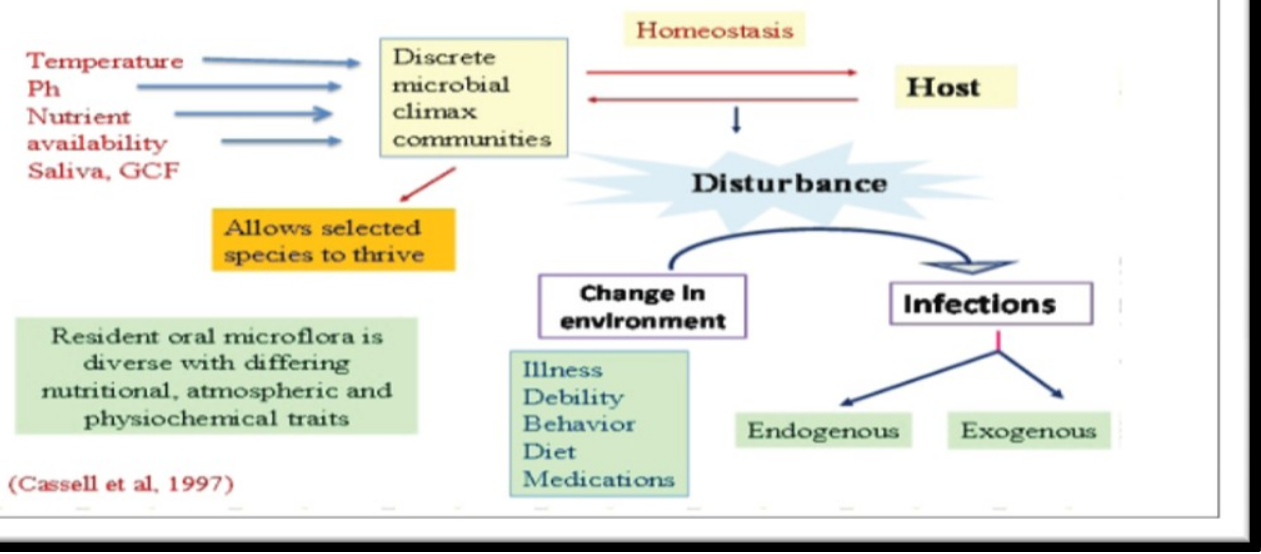

Fig 4

The oral cavity is a complex ecosystem, which has rich and diverse microbiota in it. There is a lot of metabolic activity taking place in the oral cavity (Fig 1). The wide range of $\mathrm{pH}$, nutrient availability, shedding and non shedding surfaces, salivary and crevicular fluids are the contributing elements. These fuids select localized, discrete microbial climax communities in the process of metabolic activity. ${ }^{5}$ The surprising fact is that this metabolic activity reaches a kind of homeostasis in balance with the host in spite of its fluctuation in the composition of these fluids. But most important thing is that any change in the environment disturb the homeostasis and lead to endogenous infections or susceptibility to exogenous infections (Fig 3,4)

The change in environment may be due to illness, debility, behavior, diet or medications. So, an obvious fact is that a consequence of these changes in ecology may give rise to dental diseases. ${ }^{5,6}$ (Fig4) showing oral cavity - a complex ecosystem. A dental disease is observed even with a slight change in local environment. This promotes the potential pathogens gain competitive advantage under appropriate conditions. Then, the pathogens increase in great numbers of predisposes a site to disease.

\section{Characteristics of Probiotics}

\section{Robotics And Oral Health}

Probiotics are living microorganisms, principally bacteria that are safe for consumption, which have a beneficial effect in health. To be called a probiotic, a bacterial strain must be fully characterized. The FAO and the WHO have recommended that probiotic bacterial strains are characterized by their spectrum of resistance to antibiotics and hemolytic activities, their capacity to produce toxins, their infectious power in immune compromised animal models and their side effects in humans. These probiotics strains should then be submitted to randomize clinical trials. The result of such studies should demonstrate an improvement of health ${ }^{7}$.

\section{Composition of Probiotics}

Probiotics can be yeast, bacteria or moulds. Most commonly they are bacteria. Some of these bacterial species are:

1. Lactic acid producing bacteria (LAB): Lactobacillus, Bifidobacterium, Streptococcus.

2. Non LAB species: Bacillus, Propionibacterium

3. Non pathogenic yeasts: Saccharomyces

4. Non spore forming and non flagellated rod or Coccobacilli

Probiotics have many positive influences in creating better oral health. Probiotics have both direct and indirect interactions. The advantages of direct interactions are many. Basically probiotics help in binding oral microorganisms to proteins \& biofilm formation. They fight against plaque formation and on its complex ecosystem by compromising and intervening with bacterial attachments. Through its direct interactions, probiotics compete with oral microorganisms of substances available. This process is the involvement of metabolism of substrate. Probiotics produce chemicals to inhibit oral harmful bacteria that damage oral hygiene ${ }^{8}$ .On the other hand, the indirect interactions of probiotics are effective in the process of removing harmful bacteria and stabilizing normal conditions. Probiotics modulate and systematize immune function on local community as well as non-immunologic defence mechanisms. Probiotics have the ability to regulate permeability and also to develop colonies in oral microflora with less pathogenic species. Probiotics have proved to be effective in curing diseases such as dental caries, periodontal diseases, halitosis and candidiasis. ${ }^{9,10,11,12-13}$ 
The mechanism of adhesion to oral surfaces is an issue of importance for the long-term probiotic effect of the microorganisms. Among the different assays available to study the adhesion enomenon, two model systems predominate: systems using saliva-coated hydroxylapatite, and hydroxylapatite coated with buffers, proteins and other substances. ${ }^{14}$

The pattern of adhesion of different probiotic strains to oral epithelial cells has been tested as Well. Most of the experiments on adhesion have been carried out with strains broadly used as probiotics in dairy products such as yogurt and cheese. ( $\mathrm{Tab} 2$ ) shows different means of probiotics administration for oral health purposes.

Table No.- 2 Different Means of Probiotic Administration for Oral Health Purposes

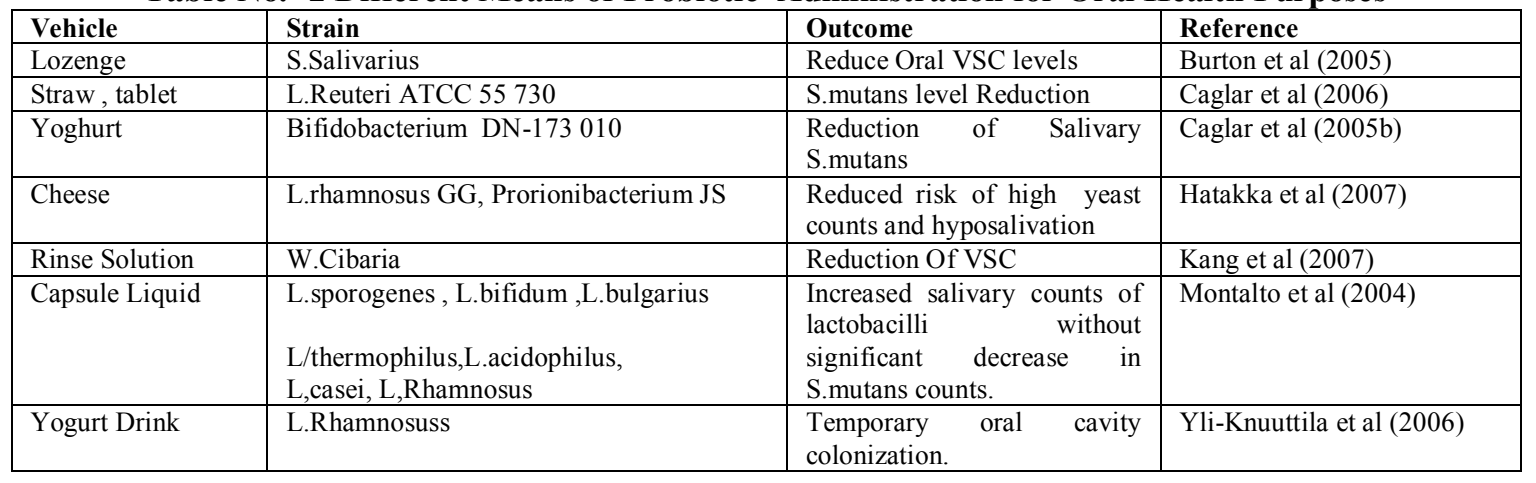

\section{Prebiotics}

Prebiotics have been proved to be an aid to complement probiotics in the treatment of oral diseases. Prebiotics are non-digestible dietary supplements. Their function is to enhance the growth and activity of beneficial organisms and simultaneously suppress the growth and activity of potentially deleterious bacteria. In this way prebiotics modify the balance of the intestinal micro-flora. The characteristic feature of prebiotic ingestion is mainly to change microbial population density.$^{15}$

Some of the commonly known prebiotics are Lactose, Inulin, Fructo oligosacccharides, Galacto oligosaccharides and Xylo oligosaccharides. Prebiotics are naturally found plenty in certain fruits like bananas, asparagus, garlic, tomato and onion wheat .The characteristic features of ideal prebiotics are as follows _ They are neither to be hydrolysed nor absorbed by mammalian enzymes or tissues. They are selectively enriched with a limited number of beneficial bacteria. The most important characteristic feature is that prebiotics can alter the intestinal micro-flora and its activities. Prebiotics can also change luminal or systemic aspects of the host defense system. ${ }^{16}$

\section{Ideal requirements of probiotic products}

1. Should show the beneficial effect on host animal.

2. Should be non-pathogenic, nontoxic.

3. Should replace and resist the intestinal micro-flora.

4. Should be capable of surviving and metabolizing in the gut environment e.g. Resistant to low $\mathrm{pH}$.

5. Should remain viable under storage for duration.

\section{Relationship between Prebiotics and Probiotics}

Prebiotics when combined with probitocs have many advantages. Basically, prebiotics selectively stimulate the growth of probiotics, which is dose and strain dependent. Prebiotics serve as a selective growth substrate for the probiotics strain during fermentation, during the period of storage, or during its passage through the gut. ${ }^{17}$ These two combinations implant live microbial dietary supplements and create a congenial environment for their survival in gut flora. Thereby this environment in gut flora improves healthy microbial balance. So, the combination of prebiotics and probiotics may have additive and synergistic effect in providing better oral health conditions.

Paster et al in an attempt to determine bacterial diversity in the human subgingival plaque by using culture-independent molecular methods have estimated that the total species diversity in the oral cavity ranges between 500 and 600 species. This number was further extended by Kazor et al, who detected 200 additional unknown species on the dorsum of the tongue, making the number of species in the mouth to reach 700.Lactobacilli make approximately $1 \%$ of the cultivable oral microflora. ${ }^{18,19}$

Definite S. mutans count reduction after a 2-week consumption of yoghurt containing L. reuteri. A temporary reduction in S. mutans was observed during the period of yogurt intake and few days after cessation 
of consumption, indicating the necessity of continual administration of the probiotic in order to achieve an effect. $^{20}$

Studies showed in a placebo-controlled randomized double-blind intervention study that the administration of probiotics lactobacilli (LGG) to kindergarten children in Helsinki, Finland, resulted in reduction of their caries risk and initial caries development. ${ }^{21}$ Certain conditions are required to remove cariogenic bacteria from the surface of the teeth to fight against dental caries. First, probiotic bacteria must be able to stick to the tooth surface where cariogenic bacteria reside. Secondly they must become a part of the biofilm that develops on teeth. Finally they must compete with cariogenic bacteria. All this process helps display a probiotic effect against carries by drastic reduction of the levels of cariogenic bacterial growth. ${ }^{21}$

\section{Probiotics and Periodontal Disease}

The study on Streptococcus oralis and Streptococcus uberis, beneficial bacteria, has proved to be useful in decreasing the growth of disease causing bacteria. Even the presences of S. oraIis and S. Uberis have proved to be a good indication of healthy gingiva.

Grudianov et al found that when compared to mouth wash Tantum Verde; selected strains of L.reuteri have reduced gingivitis\& plaque much better and have reduced the counts of S. mutans as well. ${ }^{11,12,21}$ All this is with respect to the cases of gingivitis and periodontitis Tablets containing 6.7 X 108 colony forming units (CFU) tablet of L. salivarius and Xylitol $(280 \mathrm{mg} / \mathrm{tab})$ significantly decreased the plaque index. This was proved to be effective in the case of smokers. ${ }^{22}$

\section{Mechanism of action of probiotics}

Several mechanisms have been proposed regarding action of probiotics. These bacterial strains act by secreting various antimicrobial substances such as, organic acids, hydrogen peroxide and bacteriocines. Furthermore, they compete with pathogenic agents for adhesion sites on mucosa. Probiotics can also modify the surrounding environment by modulating the $\mathrm{pH}$ and/or the oxidation reduction potential, which may compromise the ability of pathogen to become established. Finally they provide beneficial effects by stimulating nonspecific immunity and modulating the humoral and cellular immune response.

\section{Role of probiotics in general health}

Studies have shown that probiotics are used to treat various health problems such as, inflammatory bowel disease, gasrointstinal disorders, in treating antibiotic associated and traveller's diarrhea, in delaying the onset of cancer, to treat lactose intolerance and elevated cholesterol. They are also used to treat alcoholic lever diseases, ulcerative colitis, in individuals with an allergy to milk protein, asthma, hepatic encephalopathy, juvenile chronic arthritis, hypertension, urogenital infections and to aid in calcium absorption. ${ }^{3,8}$

\section{Role of probiotics in oral health Mechanism of action}

The mechanism of action of probiotics in oral health is by direct and indirect mechanisms. Probiotics bring about direct action by:

- Direct interaction on dental plaque,

- Preventing plaque formation by competing and involvement with bacterial attachment on the tooth surface.

Probiotics show indirect action by:

- Modulating the systemic immune system,

- Affecting local immunity,

- Regulation of mucosal permeability.

- Act as antioxidants and prevent plaque formation by neutralizing free electrons. ${ }^{22}$

\section{Probiotics and dental caries}

Dental caries is one of the most common oral diseases that need early prevention and intervention. Streptococus mutans is one of main causative organism for dental caries. Elevated levels of streptococcus counts are strongly associated with increased risk of dental caries. ${ }^{10}$ Studies have shown that probiotics containg L. rhamnosus GG and L. casei or Bifidobacterium DN-173 010, have significantly reduced the growth of oral streptococci and dental caries risk. ${ }^{23}$ Fermentation of glucose, fructose, mannitol, and trehalose by L. rhamnosus GG resulted in $\mathrm{pH}$ values between 5.2 and 6.8 following 24 hours of incubation, thus decreases the decalcification effect of S. mutans. It has been found that subjects without caries experience are colonized by lactobacilli that possess a significantly increased capacity to suppress the growth of S. mutans.

Certain conditions are required to remove cariogenic bacteria from the teeth surface to fight against dental caries. Firstly, probiotic bacteria must be able to stick to the tooth surface where cariogenic bacteria 
reside. Secondly, they must become a part of the biofilm that develops on teeth. Finally, they must compete with cariogenic bacteria. All this effects of probiotics helps in a drastic reduction of the levels of cariogenic bacterial growth.

\section{Periodontal health}

Various oral bacteria are related to periodontal and gingival diseases. The presence of S. oralis and S. uberis often associated with healthy gingiva. Mouth wash using selected strains of L. reuteri or tablets containing $6.7 \times 108$ colony forming units of L. salivarius and Xylitol $(280 \mathrm{mg} / \mathrm{tablet})$ has shown the reduction in gingivitis and plaque accumulation. ${ }^{5}$ Studies have shown that 14-day intake of L. reuteri led to the establishment of the strain in the oral cavity and significant reduction of gingivitis and plaque in patients with moderate to severe gingivitis. ${ }^{11}$ A L. salivarius WB21 containing tablet when administered to a test group compared with placebo demonstrated insignificant differences in pocket probing depth and bleeding on probing indices. L. casei 37 can reduce the number of most common periodontal pathogens and L. salivarius TI 2711 inhibit P. gingivalis when given for 4 or 8 weeks. ${ }^{12}$

\section{Probiotics and Halitosis}

Halitosis (bad breath) is believed to affect a large proportion of the population. It has a significant socio-economic impact and may reveal an underlying disease. Halitosis is caused by a number of volatiles, which originate from the oropharynx or from expired alveolar air. In oral malodor, the sulphur containing gases (hydrogen sulfide, methyl mercaptan and dimethyl sulfide), which are derived from the bacterial degradation of sulphur containing amino acids in the oropharynx, play a significant role. A diverse consortium of bacteria has been found to contribute to the problem, including Fusobacterium nucleatum, R gingivalis, R intermedia and Treponema denticola. Other gases, such as indole, skatole, putrescine, cadaverine and acetone, are also relevant and sometimes even the dominant cause of halitosis, although their substantively is much lower ${ }^{19}$. Most (85\%) of the pathology causing halitosis lies within the oropharynx (tongue coating, gingivitis, periodontitis, tonsillitis).

Kang et al. were the first to use a more scientifically based step-by-step approach in their quest to find a probiotic for the treatment or prevention of halitosis. ${ }^{23,24}$ ln children, halitosis has been reduced after gargling with Wcibaria containing rinse. Because of this, there has been a marked reduction in the levels of $\mathrm{H} 28$ and CHZSH by approximately $48.2 \%$ and $59.4 \%$ respectively. ${ }^{25,26}$

Studies carried out to investigate the effect of S. salivarius on oral malodour parameters. The aim was to alleviate halitosis by pre-emptively colonizing the oral cavity with a competitive commensal bacterium following a short course of mechanical and chemical treatment to reduce the numbers of odor-causing organisms and possibly provide additional attachment sites for the colonizing strain. S. salivarius was selected as an oral probiotic because it is an early colonizer of oral surfaces and is amongst the most numerically predominant members of the tongue microbiota of 'healthy' ndividuals. This species also has only a limited ability to produce volatile sulphur compounds and is unlikely to contribute significantly to oral odon S. salivarius has not been implicated either in caries or in other infectious diseases of humans and is most closely related to $\mathrm{S}$. thermophilus, a bacterium which is widely used in the dairy food industry. ${ }^{27,28,29}$

\section{Probiotics And Candidiasis}

Oral cavity with its variety of functions and complex structures is a specific site with its inherent pathology and diseases although the mouth is of course closely related to other parts and systems of the body. Candida albicans is among the most common infectious agents in the oral cavity. The incidence of yeast infections is higher at older age and under conditions of impaired immunity.

Ii takk et al were the first to perform a randomized, double-blind, placebo-controlled study on the effect of probiotics on the prevalence of oral candida. A decrease in the prevalence of C. albicans in the elderly after consumption of probiotic cheese containing L. rhamnosus GG and ropionibacterium freudenreichii ssp. shermanii IS which was as an interesting observation in this randomized placebo-controlled trial. ${ }^{30} \mathrm{~A}$ concomitant feature of the probiotic activity observed in this study was the diminished risk of hyposalivation and the feeling of dry mouth ofthe subjects lt could be hypothesized that extending research on oral pathology such as yeast infections, with respect to probiotics, and analyzing the molecular mechanisms of probiotic activity might further broaden the field of their potential applications. ${ }^{21}$

\section{Safety Issues}

The issue of protection is of particular concern during the past few years due to the amplified probiotic supplementation of different food products. From the safety point of view, the putative probiotic microorganisms should not be pathogenic, should not have any growth-stimulating effects on bacteria causing diarrhea, and should not have an ability to transfer antibiotic resistance genes. The probiotics should rather be 
able to maintain genetic stability in oral microflora. The increased probiotic utilization inevitably leads to increased concentrations of these species in the host organism. Lactobacillus bacteremia is a rare entity, and data on its clinical significance are mainly found through case reports. For the last 30 years there have been approximately 180 reported cases. ${ }^{30}$

Due to increased probiotics supplementation of different food products, safety measures are a major concern. Probiotics are often regulated as dietary supplements rather than as pharmaceutical or biological products. For the safety purpose, probiotic microorganisms should not be pathogenic, should not have any growth stimulating effects on bacteria causing diarrhoea, and should not have an ability to transfer antibiotic resistance genes, and should not cause sepsis or bacteremia. The probiotic should maintain genetic stability in oral micro-flora. ${ }^{3,12}$

Several results from antibiotic susceptibility tests claim that the tet- (W) and tet- (S) genes in some probitic Lactobacilli and Bifidobacteriastrains are responsible for sulfamethoxazole, gentamycine, polymyxin B and tetracycline resistance. These findings show the need of minimal safety evaluation during the selection of strains for probiotic use. ${ }^{31}$

Clinical characteristics of Lactobacillus bacteremia are highly variable, ranging from asymptomatic to septic shock-like symptoms. Any viable microorganism is capable of causing bacteremia, however; especially in patients with severe underlying diseases or in immunocompromised state. Nevertheless, the present literature supports the conclusion that the incidence of Lactobacillus bacteremia is unsubstantial and that all the cases where it has been registered are individuals with other systemic diseases such as diabetes, cardiovascular diseases, gastrointestinal disorders, malignancies, or organ transplant patients.

\section{Conclusion}

There is scientific evidence that specific strains of probiotics microorganisms confer benefits to the health of the host and are safe for human use. However considerable work is required to affirm the benefits of probiotics. Probiotics are, nevertheless, a new, interesting field of research in oral microbiology and oral medicine. The research is still in the initial stage. The idea of probiotics casts new light on the connections between diet and health, including oral health. The complex interplay with respect to the mechanisms of probiotics actions in the development of microbial colonies as well as oral biofilms is yet to be known. ${ }^{32,33}$

Further studies on the combined effect of different probiotics \& prebiotics should be carried out in order to authenticate the possible additive, cumulative, or competitive modes of action in the oral environment. So fan a little has been known about the possible naturally occurring resident probiotics of the mouth. In this regard, it might be interesting to conduct studies on patients with lichen planus, pemphigus vulgaris, cicatricial pemphigoid or apthous stomatitis. Probably different probiotics are needed for therapy in oral mucosal diseases as there is difference in the microbial attachment sites on the keratinized, and non keratinized epithelium. ${ }^{13,34}$ In order to assess the best means of administering probiotics, randomized controlled trails are needed. In addition, variation in the dosage for different preventive or therapeutic purposes are also to be studied carefully in order to avoid ill-effects of the species that ferment sugar and lower oral $\mathrm{pH}$ that are detrimental to the teeth. Apart from this, general safety aspects such as those related to potential invasiveness and antibiotic resistance genes must be screened. ${ }^{12,19}$ Probiotics can be used with caution in immunocompomised patients, and contraindicated in premature infants and patients with central venous access in place. Finally, possibilities to genetically modify or engineer potential probiotics strains may offer totally new visions need to studied. ${ }^{13,19,35}$

\section{Future Prospects}

Wider application of probiotics in general health can increase its demand. In future, probiotics application can be extended to cure many health related problems. The critical steps in wider application will be to make products available that are safer and clinically proven in a specific formulation, which should be easily accessible to physician and consumers. In India, sporolac i.e., Sporolactobacilli is commonly used probiotic. Recently Bacillus mesentricus is used as an alternate to B-complex. Genetically modified lactic acid bacteria have been proposed as a vehicle to deliver vaccines in the gastro-intestinal tract.

In field of oral immunology probiotics are being used as passive local immunization vehicles against dental caries. Recently by means of systemic immunization with a multivalent vaccine, L rhamnosus GG was chosen as the vehicle to harbor IgG because of its widely known health benefits in humans and animals. High titers of antibodies against human cariogenic bacteria, S. mutans and S sobrinus, were produced in bovine colostrum by a vehicle of fermented milk. ${ }^{35}$ It was found that early mucosal colonization with E. coli bacteria stimulates the mucosal immune system to produce specific antibodies as well as non-specific secretory immunoglobulins.

Such advancement will lead to the development of a new generation of probiotics, the action of which could be selected for defined disease-associated deviations in gut microbiota. This may also facilitate the potential use of genetically modified probiotics bacteria for pharmaceutical uses. Genetically modified lactic 
acid bacteria have been proposed as a vehicle to deliver vaccines in the gastro-intestinal tract. Several secretionexpression- probiotics vectors have been constructed and are currently being tested in animal models. Other probiotics carrying different immunomodulating molecules are currently being tested. Also, the probiotic vector has been modified to provide a way to deliver the active ingredient at specific targets in the gastrointestinal tract.

Various processing advances, such as microencapsulation and bacterial coating and addition of prebiotic compounds used as growth factors for probiotic organisms, will provide a means to optimize the delivery and survival of strains at the site of action in present day technology has improved drastically. Very soon, people will be able to go into space to live on the planets like the moon. NASA of USA is carrying out research to develop probiotic products which enable humans live in space. ${ }^{32,35}$ For all these valid reasons, the use of probiotics has become an emerging subject in the field of dentistry at present. Probiotics combined with prebiotics are innovative and revolutionary method in the treatment of dental diseases. So, It can be rightly said that bacteria night someday keep the dentist away.

\section{References}

[1]. Bonifeit L, Chandad F and Grenier D. Probitics for oral health: Myth or reality? JCDA. 2009;75:585-90.

[2]. Saraf K, Shashikanth MC, Priya T, Sultana N and Chaitanya NC. Probitics - Do they have role in medicine and dentistry. J Assoc Physicians India 2010;58:488-92.

[3]. Narang S, Gupta R and Narang A. Probitics in oral health care- A review. Int J Sci Eng Res 2011;2:1-5.

[4]. Grajek W, Olejnik A and Sip A. Probiotics, 'Prebiotics and antioxidants as functional foods. Acta Biochim Pol 2005;52:665-71.

[5]. Simon GL and Gorbach SL. Intestinal flora in health and disease. Gastroenterology1984; 86: 174-193.

[6]. Wijnkoop IL, Sanders M E , Cabana MD, Caglar E and Corthier G. Probiotic and Prebiotic Influence Beyond the Intestinal Tract .Nutr Rev.2007; 65 (11):469-489.

[7]. Salminen S, Boyley C, Boutron-Ruault M.C and Cummings lh. Functional food science and gastrointestinal physiology and function. Br] Nut121998; 80: 147-171.

[8]. Gronlund MM, Arvilommi H, Kero R Lehtonen and Isolauri E .Importance of intestinal colonisation in the maturation of humoral immunity in early infancy: a prospective follow up study of healthy infants aged 0-6 months. Arch Dis Child Fetal Neonatal Ed.2000; 83: 1 86-192.

[9]. Ouwehand A.C, Isolauri E and Kirjavainen PIL Salminen SI. Adhesion of four Bidobacterium strains to human intestinal mucus from subjects in different age groups. FEMS Microb Lett.1999; 172: 61-64.

[10]. Isolauri E and Kirjavainen PIL Salminen SI. Probiotics-a role in the treatment of intestinal infection and inflammation. Gut.2 002; 50(Suppl III): 54-59.

[11]. Plinius Secundus Maior G, Naturalis historiae AD and Metchnikoff E. Lactic acid as inhibiting intestinal putrefactions. In: Metchnikoff E, Mitchell PC, editors. The prolongation of life; optimistic studies. London: W.Heinemann, 1907; 161-183.

[12]. Lilly DM, Stillwell RH. Probiotics: growth-promoting factors produced by microorganisms. Science.1965; 147: 747-748.

[13]. Fuller R. Probiotics in man and animals. Appl Bacteriol.1989; 66: 365-378.

[14]. Caglar E, Cilder SK, Ergeneli S, Sandalli N and Twetman S. Salivary mutans streptococci and lactobacilli levels after ingestion of the probiotics bacteriumLactobacillus reuteri ATCC 55739 by straws or tablets. Acta OdontolScand 2006; 64: 314-318.

[15]. Caglar E, Kargul B, Tanboga I . Bacteriotherapy and probiotics' role on oral health. Oral Dis.2005a; 11: $131-137$.

[16]. Meruman I H and Stamatova I. Probiotics;Contribution To Oral Health. Oral Dis.2007; 13:443-451.

[17]. Parker R B. Probiotics, the other half of the antibiotic story. Anim Nutr Health.1974;29:4-8.

[18]. Havenaar R and I-luis Int Veld MIH. Probiotics: a general view. In: Lactic acid bacteria in health and disease. Vol.1 Amsterdam: Elsevier Applied Science Publishers, 1992.

[19]. Schaafsma G. State of art concerning probiotic strains in milk products. IDF Nutr News Lett.1996; 5: $23-24$.

[20]. Naidu AS, Bidlack WR and Clemens RA. Probiotic spectra of lactic acid bacteria (LAB). Crit Rev Food Sci Nutn1999; 39 : 13-126.

[21]. Schrezenmeir I and de Vrese M. Probiotics, prebiotics, and synbiotics: approachinga definition. Am Clin Nutr.2001; 73: 3618-3645

[22]. Teughels VIL Van Essche M, Sliepen 1 and Quirynen M.Probiotics and oral health care; periodontal 2000.2008; 48:111-147.

[23]. Corcoran BM, Ross RR Fitzgerald GF et al. Comparative survival of probiotic lactobacilli spray-dried in the presence of prebiotic substances. I Appl Microbiol.2004; 96: 1024-1039.

[24]. Meurman H. Probiotics: do they have a role in oral medicine and dentistry. Oral Sci.2005; 113(3): 188-196.

[25]. El-Nezami HS, Polychronaki NN, Ma et al .Probiotic supplementation reduces a biomarker for increased risk of liver cancer in young men from Southern China. Am] Clin Nutn2006. 83: 1199-1203.

[26]. Mego M, Majek I, Koncekova R et al.Intramucosal bacteria in colon cancer and their elimination by probiotic strain Enterococcus faecium M-74 with organic selenium. Folia Microbiol .2005; 50: 443-447.

[27]. Commane D, Hughes R, Shortt C and Rowland I .The potential mechanisms involved in the anti-carcinogenic action of probiotics. Mutat Res. 2005; 1 1:276-289.

[28]. Brzozowski Il Konturek PC, Mierzwa M et al. Effect of probiotics and triple eradication therapy on the cyclooxygenase (COX)-2 expression, apoptosis, and functional gastric mucosal impairment in Helicobacter pylori-infected Mongolian gerbils. Helicobacter. 2006; 1 1:10-20.

[29]. Bergonzelli GE, Blum S, Brussow H and Corthesy-Theulaz I .Probiotics as a treatment strategy for gastrointestinal diseases? Digestion. 2005; 72: 57-68.

[30]. Reid G, Bruce AIM Fraser N, Heinemann C, Owen I and Henning B.Oral probiotics can resolve urogenital infections. FEMS Immunol Med Microbiol ,2001;30:49-52.

[31]. Falagas ME and Betsi GI and Tokas 'II Athanasiou S. Probiotics for prevention of recurrent urinary tract infections in women: a review ofthe evidence from microbiological and clinical studies. Drugs.2006; 66: 1253-1261.

[32]. Christensen HR, Larsen CN, Kaestel P et al .Immunomodulating potential of supplementation with probiotics: a dose-response study in healthy young adults. FEMS Immunol Med Microbiol.2006; 47: 380-390.

[33]. Rinne M, Kalliomaki M, Arvilommi H, Salminen S and Isolauri E . Effect of probiotics and breastfeeding on the bifidobacterium and lactobacillus/enterococcus microbiota and humoral immune responses. I Pediatr.2005; 147:186-191.

[34]. Dent Rest(Isfahan),Probiotics for prevention of recurrent infection ,2013 May-Jun,10(3),302-306

[35]. RaoY, Lingameneni B and Reddy,Probiotic ;A role in oral Medicine and Dentistry, D. JNJ Dent Assoc,2012 Spring;83(2)28-32. 